\title{
THE CURE OF CAVERNOUS SINUS THROMBOPHLEBITIS*
}

\author{
BY \\ A. MiKLós \\ Szombathely, Hungary
}

Suppuration in the adnexa of the eye is always serious, as we can never be sure that the seemingly harmless stye may not cause a retrobulbar phlegmon, and subsequently cavernous sinus thrombophlebitis may develop on account of the rich anastomoses between the two areas. Every ophthalmologist can remember more than one case in which it was impossible to save the patient's life.

Until recently, the different antibodies, e.g., intravenous hexamethylenetetramine, trypaflavin, etc., have been of no avail, nor were the sulphonamides a success, so that we were absolutely helpless when faced with this condition. The discovery of penicillin, however, has solved the problem, as it seems that the disease can be cured, if penicillin in adequate concentration can reach the infected areas by way of the spinal fluid.

We know of so few cases reported in the literature that we feel justified in supposing that the therapy of this illness has not yet been standardized. I think it may be useful, therefore, to publish two cases which we were able to cure.

\section{Case 1.}

H. J., a boy, 3 years of age, was brought to our department on August 12, 1947. The patient's mother said that the child had had measles a fortnight before; two small papules remained on his right lower lid. On the morning of the day before the child was brought to the hospital, his right upper lid began to swell. In the evening this swelling became worse and the patient developed a high temperature.

When the child was admitted to hospital both lids of his right eye were red and very swollen and the protrusion was $1-1.5 \mathrm{~cm}$. The boy was somnolent and had a temperature of $104^{\circ} \mathrm{F}$. On the left side a moderate oedema of the lids was visible, the other parts of the eye were normal. The back of the neck was slightly stiff, the spinal fluid faintly cloudy, the number of cells was 100-120. The diagnosis was retrobulbar phlegmon of the right side, incipient cavernous sinus thrombophlebitis.

The treatment consisted of a moist dressing, 4 tablets of sulfa-methylthyasol daily, and the intramuscular administration of 20,000 units of penicillin every three hours.

On the following morning the patient's temperature had dropped to $98.5^{\circ} \mathrm{F}$, the oedema of the left eye-lid was almost completely gone and the child was much more lively. The blood picture only showed an increase in the leucocyte count $(12,000)$. The rhinological examination revealed a very small amount of purulent secretion on the posterior end of the middle concha, so we resolved to continue the penicillin treatment and to remove the ethmoidal cells. This offered no difficulty.

Until August 17 we carried on the systematic penicillin treatment and up to that day (the 5 th) the patient had been given 1,200,000 units intramuscularly. His

\footnotetext{
* Received for publication March 11, 1949.
} 
temperature became normal and the exophthalmos of the right eye began to recede. Then the penicillin treatment was stopped.

On August 18 the patient became feverish and his temperature rose to $104^{\circ} \mathrm{F}$., the exophthalmos became worse and he grew extremely restless. We gave him 20,000 units of penicillin every three hours intramuscularly and a blood transfusion of $100 \mathrm{cc}$. daily.

On August 20 the patient's condition improved, his temperature was normal; the exophthalmos receded slightly; in the centre of the cornea a small abscess had developed, but healed perfectly in a few days with local treatment.

On August 26 the exophthalmos had considerably diminished; the child was so lively that he could hardly be kept in bed; the temperature had been normal for five days. The spinal fluid was only faintly opalescent, cells 10-13. We stopped further treatment.

On September 5 the child left the hospital. The right upper eye-lid was still a little. swollen with slight ptosis. No exophthalmos. In the centre of the cornea a faint macula was visible. The fundus was in perfect condition. Visual acuity could not be exactly determined, but was apparently good.

At a re-examination on November 2, apart from persistent drooping of the upper lid of the right eye $(4 \mathrm{~mm}$.) and a small paracentral macula the size of a pinhead, the eyes were in good condition.

On November 21,1948, we enquired after the little boy's condition and were told by his mother that he was perfectly well, that he was developing splendidly, and that the right eye-lid was no longer drooping.

\section{Case 2.}

N. 1., a girl aged 18 years, applied for admission to the hospital on May 19, 1948. Her father said that three weeks previously a furuncle had developed on the right side of the girl's forehead, which had healed in a few days after having been opened by a doctor. 'Ten days before the girl was admitted to the hospital, another furuncle had developed underneath the first one; the doctor again opened and drained it, and prescribed two tablets of sulfamethylthyasol four times daily. In spite of this, the girl's condition did not improve, she became feverish and her right upper eye-lid started swelling.

The patient's condition when admitted to the hospital on May 19, 10 a.m.: a rather thin, languid girl, perfectly conscious; temperature $102 \cdot 2^{\circ} \mathrm{F}$, , pulse full.

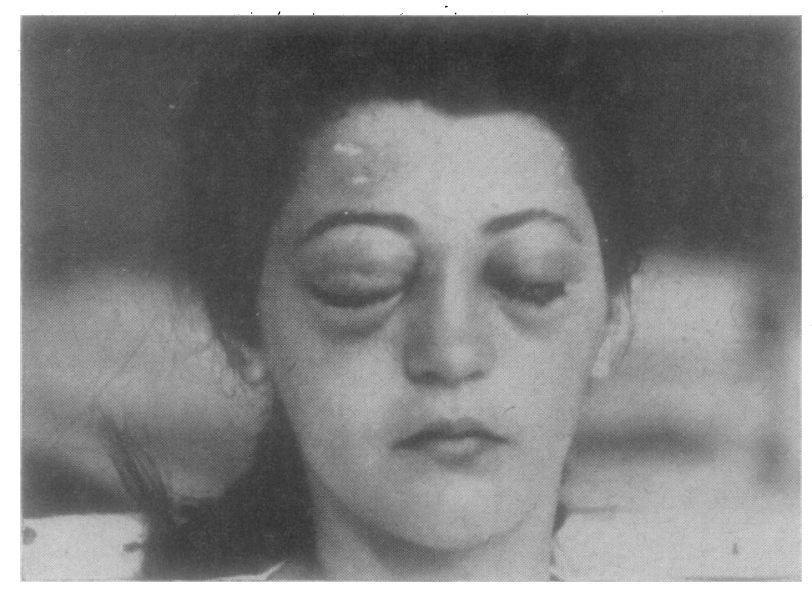

FIG. 1.-Thrombophlebitis of the cavernous sinus fully developed. The two incisions on the right side of forehead clearly shown. 
slightly accelerated. V.A. right eye $5 / 10$, left eye $5 / 8$. The lids of the right eye were considerably swollen and bluish. Conjunctiva chemotic to a high degree, veins filled, tortuous. Exophthalmos about $1.5 \mathrm{~cm}$. Normal fundus. On the left side only a slight swelling of the eye-lids was visible. The diagnosis was retrobulbar phlegmon, incipient cavernous sinus thrombophlebitis.

We immediately started penicillin treatment-20,000 units every three hours intramuscularly - as well as 2 tablets hexamethyl-enetetramine four times daily and a moist dressing locally. At 5 p.m., when the patient had already been given 3 doses of 20,000 units of penicillin, her condition became worse. The exophthalmos of the right eye increased, and a similar condition began to develop on the left side. The patient became somnolent and her temperature rose to $103^{\circ} \mathrm{F}$. We were faced with the classical symptoms of thrombophlebitis of the cavernous sinus. At 9 p.m. the patient was completely unconscious, the back of her neck was rigid, the exophthalmos of the right eye was about $2 \mathrm{~cm}$. and that of the left eye $1.5 \mathrm{~cm}$.

We decided to administer penicillin intracisternally. After withdrawing $40 \mathrm{cc}$. of slightly cloudy spinal fluid which was under great pressure, we injected 20,000 units dissolved in $2 \mathrm{cc}$. of normal saline into the cisterna cerebellomedullaris.

Report on spinal fluid: albumen $120 \mathrm{mg}$. per cent. Pándy ++ , Nonne-Apelt + , number of cells : 960 per mm., bacteriologically negative.

We prescribed 20,000 units of penicillin twice daily intracisternally, and 30,000 units of penicillin intramuscularly.

Next day patient was conscious and feeling better. The exophthalmos somewhat less, temperature $102^{\circ} \mathrm{F}$. Therapy the same as before, but the patient was given, in addition, a daily blood transfusion of $100 \mathrm{cc}$.

After the fourth intracisternal injection of penicillin the report on the spinal fluid was: pressure reduced, cell count 312 per cc., albumen $140 \mathrm{mg}$. per cent., Pándy + , Nonne-Apelt + , sugar $0.4 \mathrm{mg}$. per cent.

On May 21, the third day of the treatment, the improvement was considerable, temperature $100.4^{\circ} \mathrm{F}$. The intramuscular administration of penicillin was continued, but the intrathecal injections of 20,000 units were reduced to one daily. A blood transfusion of $100 \mathrm{cc}$. was continued daily.

May 26. The exophthalmos was definitely less pronounced on both sides. The patient opened her eyes spontaneously, her temperature was $97.7^{\circ} \mathrm{F}$., her appetite was returning. The spinal fluid flowed with normal pressure. Cell count 100 per cc., Pándy + . Therapy as before.

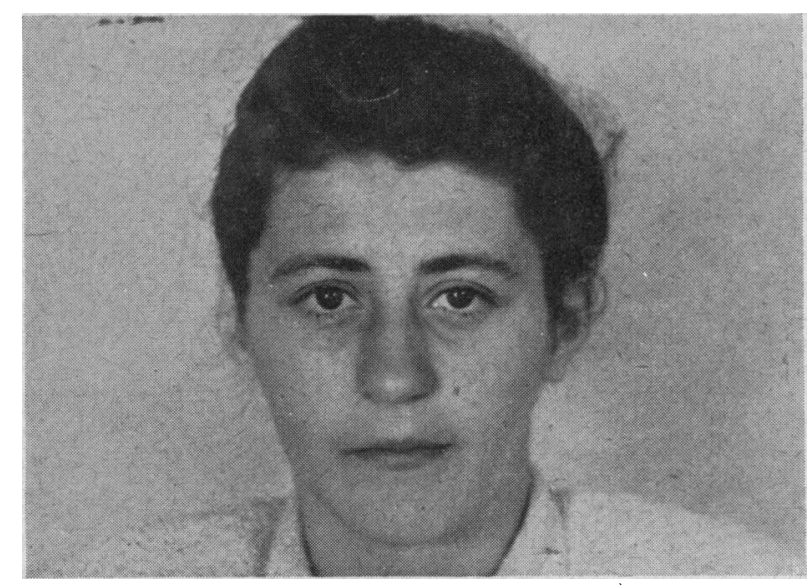


May 28. The patient perfectly well. Report on spinal fluid: cell count 40 per cc., sugar $38 \mathrm{mg}$. per cent., albumen $20 \mathrm{mg}$. per cent., Pándy 0 , NonneApelt 0 . No further treatment.

June 5. The patient left the hospital. No exophthalmos, both optic discs slightly hyperaemic. The right eye showed a $15^{\circ}$ convergent squint. V.A. in both eyes $5 / 5$.

September 27. Control examination. The girl was feeling well, had considerably increased in weight, and could carry on her normal work. The squint was better, V.A. in both eyes $5 / 5$.

In the first case penicillin was administered in the usual way, intramuscularly, while in the second case systemic and intrathecal treatment were combined. In the first case intramuscular treatment gave satisfactory results, but in the second case, despite this method of administration of penicillin the patient developed thrombophlebitis of the cavernous sinus and, in consequence, incipient purulent meningitis.

What was the cause of this failure?

As Friedemann (1931), Elkeles (1947), and others have ascertained, the transfer of certain substances from the blood to the spinal fluid depends on the negative electric charge of these substances and on their concentration in the blood. Inder normal circumstances penicillin which has a negative charge can reach the spinal fluid when adequate concentrations are present in the blood through the bloodbrain barrier. These authors have also proved that when the meninges are inflamed their permeability generally increases.

Barton and others (1947), however, have found that with a normally permeable blood-brain barrier the usual 20,000 to 50,000 units of penicillin administered intramuscularly are not detectable in the spinal fluid, and that if the desired bacteriostatic concentration is to be attained 600,000 to $1,000,000$ units must be given intramuscularly.

Views differ as to the question of permeability in meningitis, and thus opinions vary as to the quantity to be administered intramuscularly to produce an efficacious concentration in the spinal fluid. According to the latest data we may conclude that most of the authors think that intramuscular administration of penicillin alone is to be used only in the mildest cases of meningitis. In more severe cases, when coma has already set in, they consider it absolutely necessary to combine systemic and intrathecal treatment, as only in this way is it possible to ensure the required level of penicillin in the spinal fluid. This is proved by the wide experience of Mérei (1948) with purulent meningitis; also by two fatal cases of thrombosis of the cavernous sinus of De Jaeger and De Haene (1947), and by our second case, in which the patient became steadily worse in spite of systemic treatment, and in which 
recovery was only brought about by combined intramuscular and intraspinal treatment.

The question arises whether, with intrathecal treatment, penicillin should be given intracisternally or by lumbar injection. There is no doubt that the puncture of the cistern is much easier for both doctor and patient because of its simple technique. It imust also be admitted that penicillin which reaches the cisterna cerebello-medullaris is more efficacious than that given by lumbar puncture. (White and others, 1945). Apart from this we have to consider the possibility of an obstruction of the spinal canal, a complication which does not matter when penicillin is administered by intracisternal puncture. This method, however, carries with it some danger because of the risk of restlessness on the part of the patient. Therefore most doctors resort to a lumbar puncture when there is no immediate danger of a spinal block.

According to the published data a daily dosage of 20,000 to 40,000 units administered intrathecally is the quantity required to produce a satisfactory bacteriostasis in the spinal fluid, without causing great inconvenience to the patient. Too large quantities are apt to cause headache, vomiting, and paralysis. They must therefore be considered harmful.

Blocking of the spinal canal may be caused either by obturation or by adhesions. It is characteristic of the former that when a lumbar puncture is performed the spinal fluid is dense and sticky and flows slowly, and the spinal fluid is so viscous that it may impair the circulation or even stop it. An obturation block occurs at the onset of meningitis; the adhesion block, due to fibrin, tends to occur at a later stage, during the process of recovery. With thrombophlebitis of the cavernous sinus, patients usually get adequate medical treatment at an early stage of the illness because the rapidly developing exophthalmos causes anxiety to the patient's family; therefore purulent meningitis is forestalled by treatment. In these cases there is no danger of an obturation block.

Both our patients were given microtransfusions. As is known, their object is to bring immune substances, active haenoglobin, white and red blood-corpuscles, and serum albumen to the site of sepsis. By these transfusions we also stimulate the reticuloendothelial system to produce more antibodies and to change the pathological cytogenesis of the haemopoietic system. Naturally we used the transfusions only as an indirect aid simultaneously with daily penicillin treatment until cessation of fever.

What is the correct treatment for thrombophlebitis of the cavernous sinus? If the illness is still in the early stage, which can easily be ascertained by its well-known signs and symptoms, 
and if the spinal fluid is clear or faintly opalescent with a cell count from 200 to 400 , we can try intramuscular penicillin treatment alone. If, however, the patient has already developed the classic symptoms of thrombophlebitis of the cavernous sinus, only a combined systemic and intrathecal penicillin treatment can be successful. The examination of the spinal fluid then shows a cell count of 1,000 cubic mm. or more, but is bacteriologically negative. A further question is whether penicillin should be administered by the intracisternal or lumbar route? In our opinion, in grave cases the treatment ought to begin with cisternal punctures and change over to lumbar punctures after the condition has become less grave. We resorted to this method, bearing in mind that, if possible, the less dangerous method must be chosen. Penicillin is certainly more efficacious when adminstered by intracisternal injection than by the intralumbar route. (White and others, 1945.) The effect is increased by the fact that penicillin administered by intracisternal injection is eliminated much more slowly than that given by the intralumbar route, so the bacteriostatic level of penicillin in the spinal fluid is maintained for a longer time. In view of the risks of intracisternal punctures in patients who do not keep absolutely still, we think that these should only be carried out in the first stage of a serious case.

We give below our dosage of intrathecally administered penicillin. We injected 20,000 units twice daily into the spinal fluid as long as the serious symptoms persisted, later only 20,000 units once daily. This corresponds to the data given in the literature, most authors considering 20,000 to 30,000 units daily the correct dosage with meningitis. As, however, according to Mérei (1948), normal saline, when injected into the spinal fluid, may cause headache, convulsions, etc., it is advisable to administer penicillin by dissolving 10,000 units in $1 \mathrm{cc}$. normal saline, further diluted with the spinal fluid in the syringe.

It is also very important to know when penicillin treatment should be stopped. De Jaeger and De Haene believe that in fatal cases penicillin treatment was stopped too early. We observed this in our first case. When, however, after a relapse, we restarted energetic penicillin treatment the patient recovered. Penicillin treatment is discontinued when the report on the spinal fluid shows a reduction in cell count. It should not be discontinued before the cell count is below 100. Then, naturally, the patient has had no fever for several days, and the eye symptoms are subsiding.

Besides the general treatment mentioned above and a daily microtransfusion of $100 \mathrm{cc}$, we gave the patients sulphonamides in short shocks. It is important to supply the need for vitamin $\mathrm{C}$ 
and fluids. A protective bandage should be applied to the eyes in order to avoid abscesses of the cornea which may develop while the patient is unconscious.

Thus it is evident that the treatment of thrombophlebitis of the cavernous sinus is a serious task for the ophthalmologist. He can only carry it out by most careful observation and efficient cooperation with other specialists. If treatment is begun in time this disease is now curable without leaving any traces, although up to the present time every case of thrombophlebitis of the cavernous sinus has ended fatally.

\section{Summary}

Thrombophlebitis of the cavernous sinus, which has always been fatal hitherto, is curable by a combined systemic and intrathecal penicillin treatment.

We believe that systemic treatment alone should only be attempted at an early stage of the illness, when the cell count in the spinal fluid is from 200 to 400 . In more advanced cases, only a combined treatment will give satisfactory results.

Although intracisternal injections are much more effective than intralumbar treatment, they should only be resorted to at the bèginning of severe cases (with unconsciousness, etc,) because of the patient's restlessness. When, however, the grave symptoms have disappeared, intralumbar treatment should be given.

The dose of penicillin to be injected into the spinal fluid should be 20,000 units twice daily, 10,000 units penicillin dissolved in 1 cc. normal saline which must be further diluted with the spinal fluid contained in the syringe.

Penicillin treatment ought to be stopped only when the cell count in the spinal fluid is below 100 .

Besides the intrathecal treatment, 20,000 units of penicillin are given intramuscularly every three hours.

Apart from penicillin treatment a blood transfusion of $100 \mathrm{cc}$. is given daily, and the resisting power of the body is supported in every possible way.

\section{REFERENCES}

Barton, R. L., Marshak, L., Bauer, T. J., and Loewe, L. (1947). Amer. J med. Sci., 214, 50 .

Elkeles, A. (1947). Lancei, 1, 764.

Friedeman, U. and ElKeles, A. (1931). Dtsch. med. Wschr., 57, 1934.

JAEger, A. DE and HAENE, A. DE (1947). Bull. Soc. belge Ophtal., 87, 104.

Mérei, L. (1948). Orv Lapja, 4, 1073.

VAsiutinsky, A. G. (1947). Excerpta Med., Section XII, 1, No. 3, 135.

White, W. L., MuRPhy, F. D., LOckWOOD, J. S., and FlipPiN, H. F. (1945). Amer. J. med. Sci., 210, 1. 